\title{
Improving the efficiency of hybrid combination preparation in rice breeding by a modified flowering stimulant
}

\author{
Ling Zheng ${ }^{1,3 \#}$, Shangxing Zhang ${ }^{4 \#}$, Fengyin Xue ${ }^{1 \#}$, Yifan Yun ${ }^{1}$, Pin Liu ${ }^{1}$, Hua Yuan ${ }^{1,2}$, \\ Weilan Chen ${ }^{1}$, Peng Qin ${ }^{1,2}$, Yuping Wang ${ }^{1}$, Bingtian $\mathrm{Ma}^{1}$, Shigui $\mathrm{Li}^{1,2}$, Yong Chen ${ }^{3 *}$, Bin $\mathrm{Tu}^{1,2^{*}}$ \\ (1. Rice Research Institute of Sichuan Agricultural University, Chengdu 611130, China; \\ 2. State Key Laboratory of Crop Gene Exploration and Utilization in Southwest China, Chengdu 611130, China; \\ 3. Hybrid Rice Research Center of Neijiang Academy of Agricultural, Neijiang 641000, Sichuan, China; \\ 4. Zunyi Agricultural Science Research Institute, Zunyi 563000, Guizhou, China)
}

\begin{abstract}
The utilization of hybrid vigor is an important breakthrough in the history of rice breeding. To select the best hybrid combinations, breeders manually perform extensive testing of hybridizations between restorer and sterile lines, which is a laborious and time-consuming process. Here, we report that a modified flowering stimulant containing methyl jasmonate (MeJA), 6-benzylamine adenine and kinetin effectively promotes the flowering and seed set of male-sterile rice lines. Different concentrations of the ingredients were tested to identify an optimal formulation. Seed quality evaluation indicated that hybrid seeds from plants sprayed with the flowering stimulant had a higher germination rate than seeds from plants prepared by glume-cutting. In summary, the modified flowering stimulant described in this study may help reduce the labor requirement associated with hybrid rice breeding and improve yield and efficiency.
\end{abstract}

Keywords: hybrid rice, cross, flowering stimulant, methyl jasmonate, kinetin

DOI: $10.25165 /$ j.ijabe.20201303.5632

Citation: Zheng L, Zhang S X, Xue F Y, Yun Y F, Liu P, Yuan H, et al. Improving the efficiency of hybrid combination preparation in rice breeding by a modified flowering stimulant. Int J Agric \& Biol Eng, 2020; 13(3): 36-40.

\section{Introduction}

Rice (Oryza sativa L.) is one of the most important food crops worldwide and a staple food for more than half of the world's population. Breeding of high-yielding rice is crucial for meeting the food demand of the increasing world population and increasing consumption $^{[1,2]}$. In the past several decades, semi-dwarf breeding and the utilization of heterosis have markedly improved rice yield ${ }^{[3]}$. Hybrid rice is mainly based on the three-line and two-line male-sterile systems. The three-line male-sterile system is comprised of a male-sterile line, maintainer line and restorer line.

Received date: 2019-01-03 Accepted date: 2020-04-21

Biographies: Ling Zheng, PhD candidate, research interests: hybrid rice breeding, Email: zl86311@163.com; Shangxing Zhang, Associate Professor, research interests: rice genetics and breeding, Email: zynbzsx@163.com; Fengyin Xue, Master student, research interests: genetics mechanism of rice quality, Email: 1057909155@qq.com; Yifan Yun, Master student, research interests: molecular design breeding, Email: 13016208822@163.com; Pin Liu, Master student, research interests: genetics mechanism of rice quality, Email: 15982251900@163.com; Hua Yuan, Assistant Researcher, research interests: rice grain development, Email: yuanhua1028@foamail.com; Weilan Chen, Assistant Researcher, research interests: rice heavy metal accumulation, Email: lightstop@163.com; Peng Qin, Professor, research interests: rice grain development and resource collection. Email: qinpeng626@gmail.com; Yuping Wang, Professor, research interests: hybrid rice breeding, Email: wypscwj@126.com; Bingtian Ma, Professor, research interests: hybrid rice breeding, Email: btma02@sicau.edu.cn; Shigui Li, Professor, research interests: hybrid rice breeding, Email: lishigui@sicau.edu.cn.

\# These authors contributed equally to this work.

*Corresponding author: Yong Chen, Professor, research interests: rice genetics and breeding, Email: chenyongnj@163.com; Bin Tu, Associate professor, research interests: genetics mechanism of rice quality, Mailing address: 211\# Huiming Road, Wenjiang District, Chengdu 611130, China. Tel: +86-13808189278, Email: bintu-1986@hotmail.com.
In hybrid rice varieties, the heterozygous first filial (F1) generation exhibits a yield advantage of $10 \%-20 \%$ over the inbred parental lines ${ }^{[4,5]}$. In the process of rice breeding, elite hybrid rice varieties are identified by testing thousands of hybridizations between restorer and sterile lines. The conventional hybridization method requires breeders to manually cut the spikelet glumes to pollinate male-sterile lines, which is laborious and time consuming. To overcome these disadvantages and improve efficiency, breeders have developed several treatment methods. $\mathrm{CO}_{2}$ can efficiently induce the flowering of male-sterile lines in a short amount of time, and this strategy has been utilized in rice hybridization ${ }^{[6,7]}$. However, it cannot be applied in paddy fields due to equipment limitations. Jasmonate (JA) and methyl jasmonate (MeJA) are well recognized lipid-derived stress hormones that function in a remarkable number of plant developmental events, including primary root growth, reproductive development and leaf senescence $^{[8,9]}$. Previous research indicated that MeJA can induce rice floret opening within approximately $30 \mathrm{~min}^{[10]}$, and other studies made important advancements in the application of MeJA to induce substantial and rapid flowering of male-sterile lines ${ }^{[11,12]}$. Although MeJA application obviously improves the efficiency of rice hybridization ${ }^{[10]}$, MeJA is an abiotic stress-related phytohormone with an important role in drought-induced grain yield reduction ${ }^{[13]}$. As such, exogenous MeJA causes seeds to fall off from the panicle after pollination, which strongly affects the quantity and quality of hybrid seeds. In this study, an optimal flowering stimulant formulation was identified to address the problem of grain falling associated with exogenous MeJA application in rice. Specifically, 6-benzylamine adenine (6-BA) and kinetin (KT) were incorporated into the formulation of the flowering stimulant to mitigate the grain-shattering effect of MeJA. 
In brief, application of this modified flowering stimulant in hybrid rice breeding may reduce grain shattering and help ensure high hybrid seed quality and high work efficiency.

\section{Materials and methods}

\subsection{Plant materials and growth conditions}

In this study, the flowering stimulant solutions were tested using a typical thermo-sensitive genic male-sterile (TGMS) line, Jin-4155S (Hunan Yahua Seed Industry Research Institute, China), and five cytoplasmic male-sterile (CMS) lines: Chuankang-606A (Sichuan Academy of Agricultural Sciences, China), Quan-9311A (Quanyin Hi-Tech Seed Industry Co. Ltd., Anhui, China), Yixiang-1A (Yibin Academy of Agricultural Sciences, China), Qingxiang-1A (Yufeng Seed Industry Co., Ltd., Sichuan, China) and Chuannong-1A (cultivated by our group). All plants were grown in the paddy fields of the Rice Research Institute of Sichuan Agricultural University (Chengdu, China) or Lingshui (Hainan Province, China) during the natural growing season.

\subsection{Flowering stimulant preparation}

Stock solution preparation: Tween-20 (20\% V/V) was prepared by diluting pure Tween-20 by 5 times with distilled water, Tween-20 (20\% V/V) solution storage at room temperature; 6-BA $(1 \mathrm{mg} / \mathrm{mL})$ and $\mathrm{KT}(1 \mathrm{mg} / \mathrm{mL})$ were prepared by dissolving $100 \mathrm{mg}$ regent to $10 \mathrm{~mL}$ distilled water contains few drops of hydrochloric and increasing the volume of the solution to $100 \mathrm{~mL}$ after dissolution, stock solutions were keep at $4^{\circ} \mathrm{C}$. The different stimulant formulations were obtained by diluting the stock solutions to the desired final concentrations. It should be noted that MeJA (4235 mmol/L, $1 \mathrm{~g} / \mathrm{mL}$ ) is insoluble in water, equal or double volumes of ethyl alcohol was mixed with MeJA as cosolvent before mixing with other stock solutions. Finally, the flowering stimulant solutions were mixed uniformly by hand or by vortexing.

\subsection{Application of the flowering stimulant}

The following steps were performed to apply the flowering stimulant:

(1) Panicle selection and trimming. For each of the male-sterile lines, mature panicles breaking out of the leaf sheath were selected. Additionally, it was important to select panicles in which the upper $1 / 3$ to $1 / 4$ of the spikelets had naturally flowered. These flowered spikelets were cut off with scissors, and the remaining branches were evenly dispersed by shaking to ensure that the flowering stimulant could be uniformly sprayed onto the surface of the remaining spikelets.

(2) Spraying of the flowering stimulant. The flowering stimulant solutions were sprayed before flowering in the morning or after flowering in the afternoon. After spraying the stimulant onto the surface of the rice panicles and the hybrid, paper bags were used to pack the panicles to prevent contact with pollens from adjacent plants. The recommended volume of flowering stimulant was 0.5-1.0 mL per bag.

(3) Pollination. The spikelets typically flowered 20-40 min after being sprayed the flowering stimulant in the morning. Standard methods were used to collect the paternal pollen for pollination.

\section{Results}

\subsection{Evaluation of different flowering stimulant solutions}

Four flowering stimulant solutions were tested in this study

(Table 1). First, we assessed the ability of the different solutions to induce flowering in CMS lines Quan-9311A and Chuankang-606A. All four flowering stimulant solutions rapidly and effectively induced spikelet flowering, confirming that MeJA as an active ingredient can independently induce spikelet flowering (Figure 1a, Figure S1A). Grain shattering was observed in panicles sprayed with solutions I and III, and it occurred to a greater extent in panicles sprayed with solution I (Figure 1b, Figure S1B). The high concentration of exogenous MeJA in solution III led to low seed set (Figure 1c, Figure S1C). Accordingly, the panicles sprayed with solutions II and IV had higher seed set and lower grain shattering. The thousand fresh seed weight (TFSW) of the hybrid seeds was measured $15 \mathrm{~d}$ after fertilization (DAF). Compared to the hybrid seeds from plants prepared by glume-cutting, the hybrid seeds from plants sprayed with the flowering stimulant solutions were heavier and well filled. However, solutions II and IV produced the heaviest and/or greatest number of hybrid seeds, demonstrating that these formulations are more suitable for hybrid seed production in hybrid rice breeding (Figure 1d and 1e, Figure S1D and S1E). Considering the cost and efficacy of the four different flowering stimulants, solution II was identified as the most economical and efficient for application in hybrid rice crosses.

Table 1 Contents of the flowering stimulant solutions

\begin{tabular}{ccccc}
\hline Solution & $\mathrm{MeJA} / \mathrm{mmol} \cdot \mathrm{L}^{-1}$ & Tween-20/\%(V/V) & 6-BA $/ \mathrm{mg} \cdot \mathrm{L}^{-1}$ & $\mathrm{KT} / \mathrm{mg} \cdot \mathrm{L}^{-1}$ \\
\hline I (CK) & 1 & 0.01 & 0 & 0 \\
II & 1 & 0.01 & 1 & 1 \\
III & 4 & 0.01 & 1 & 1 \\
IV & 1 & 0.01 & 5 & 5 \\
\hline
\end{tabular}

3.2 Application of the modified flowering stimulant in different male-sterile lines

Two distinct male-sterile systems are utilized in hybrid rice production: cytoplasmic male sterility (CMS) and environmentconditioned genic male sterility (EGMS) ${ }^{[14,15]}$. Wild-abortive-type (WA)-CMS lines are the most widely used lines. EGMS lines include the photoperiod- and thermo-sensitive genic male-sterile (PGMS and TGMS) lines ${ }^{[16,17]}$. The following analyses of different male-sterile lines were performed using solution II, hereafter referred to as the modified flowering stimulant. To assess its broader applicability, the effects of the modified flowering stimulant were analyzed in different types of male-sterile lines: a typical TGMS line (Jin4155S) and three distinct CMS lines (Qingxiang-1A, Yixiang-1A and Chuannong-1A). In each of these lines, flowering was effectively induced by the modified flowering stimulant within $30 \mathrm{~min}$ after spraying (Figure 2a to 2c). Interestingly, we found that it was optimal to select panicles in which the upper $1 / 3$ to $1 / 4$ of spikelets had flowered naturally, regardless of how much of the panicle had emerged from the leaf sheath. Otherwise, the flowering stimulant failed to effectively induce the spikelets to open. The selection of panicles with some natural spikelet flowering was particularly critical in Chuannong1A. If a fully emerged panicle without natural flowering was used, almost no spikelets were induced after spraying with the flowering stimulant. In contrast, approximately $90 \%$ of the spikelets could be quickly induced if panicles with some natural flowering (upper $1 / 3$ to $1 / 4$ of spikelets) were selected (Figure S2).

\subsection{Application of the modified flowering stimulant produces high quality hybrid seeds}

To investigate the effect of the flowering stimulant on seed quality, we compared the appearance and germination rates of 
hybrid seeds from plants prepared by glume-cutting (i.e., glumes manually cut with scissors) and plants sprayed with the modified flowering stimulant. The elite hybrid restorer line Shuhui498 (R498) ${ }^{[18]}$ and CMS line Chuannong1A were used for this analysis. As shown in Figure 3a and Figure 3b, the hybrid seeds from the manually prepared glume-cut plants were cone-shaped and infected with fungi (Figure 3a). In comparison, the plants sprayed with the flowering stimulant produced plump and light-colored hybrid seeds
(Figure 3b). After harvesting and drying the seeds, the germination rate of the hybrid seeds from the sprayed plants was approximately $85 \%$ (Figure $3 \mathrm{~d}$ and 3 e). In contrast, only $50 \%$ to $60 \%$ of the hybrid seeds from the glume-cut plants germinated under the same conditions (Figure $3 c$ and $3 e$ ). In conclusion, the hybrid seeds from plants sprayed with the flowering stimulant had a higher germination rate and better quality than the seeds from glume-cut plants.
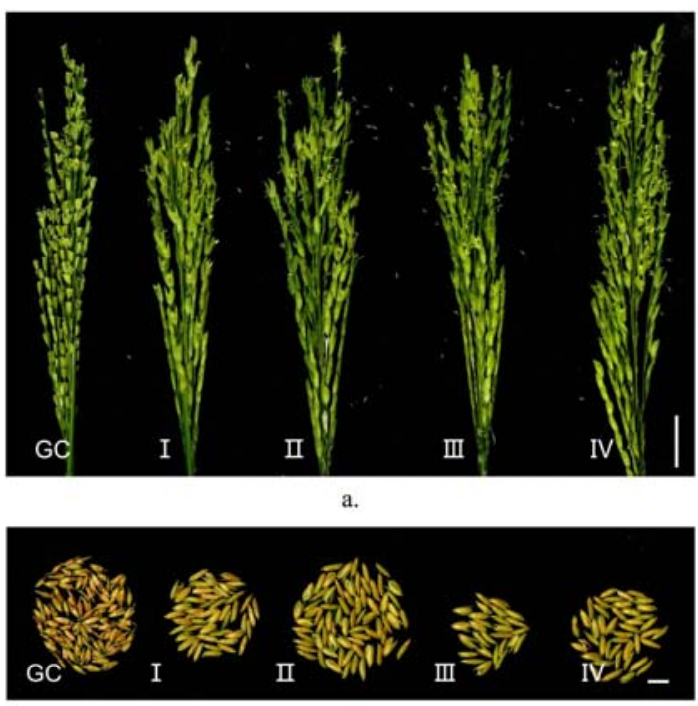

c.

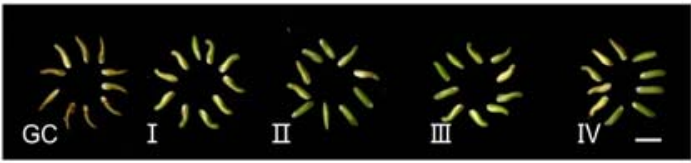

d.

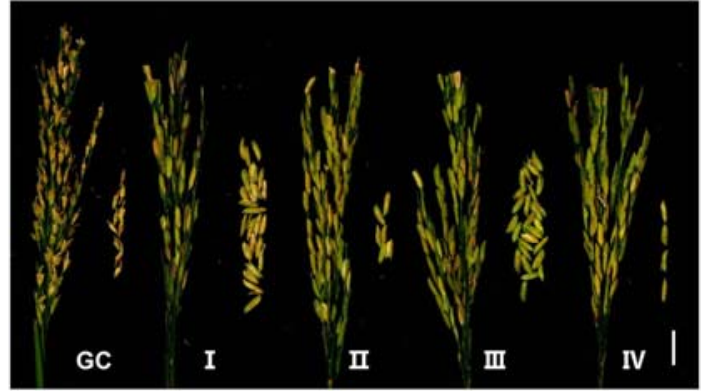

b.

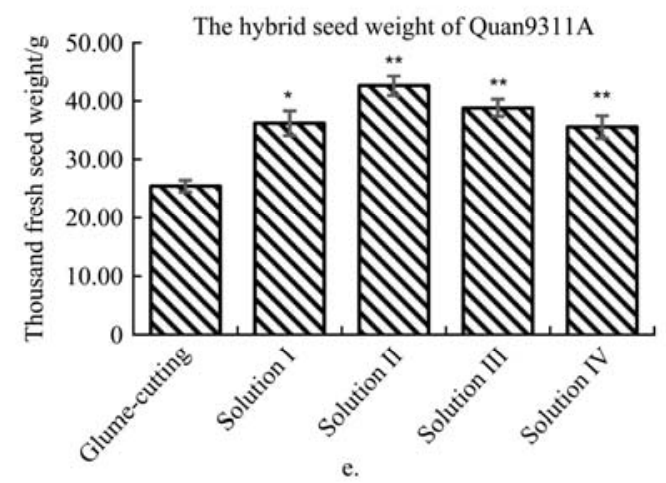

Note: (a) Spikelet flowering of Quan-9311A panicles after glume-cutting (GC) preparation or spraying with stimulant solutions I, II, III and IV. (b) Comparison of panicles and the hybrid seeds 15 days after fertilization (DAF). Spraying with MeJA alone (solution I) or a high concentration of MeJA (solution III) resulted in more severe grain shattering. (c) The hybrid seed number per panicle after glume-cutting or spraying with the flowering stimulant solutions. The application of solution II resulted in the largest number of hybrid seeds. (d) Comparison of 15 DAF caryopses. (e) Thousand fresh seed weight of hybrid seeds after glume-cutting preparation or spraying with the flowering stimulant solutions. The caryopses resulting from treatment with the flowering stimulant solutions were well filled. Scale bars: $20 \mathrm{~mm}$ in (a); $5 \mathrm{~mm}$ in (b); $10 \mathrm{~mm}$ in (c-d). Values in (e) are means \pm SD ( $n=3$ ).

Figure 1 Effects of the flowering stimulant solutions on grain shattering and seed set in CMS line Quan-9311A

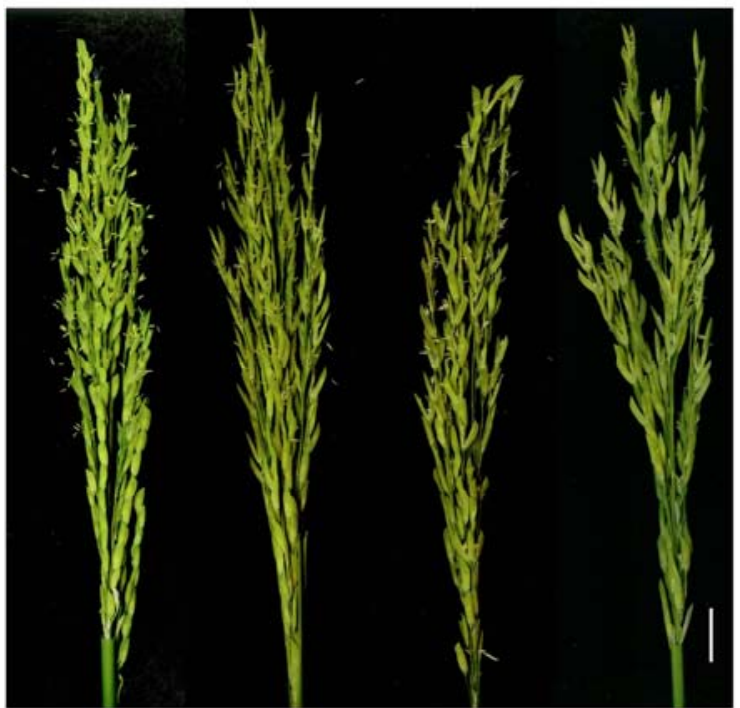

a.

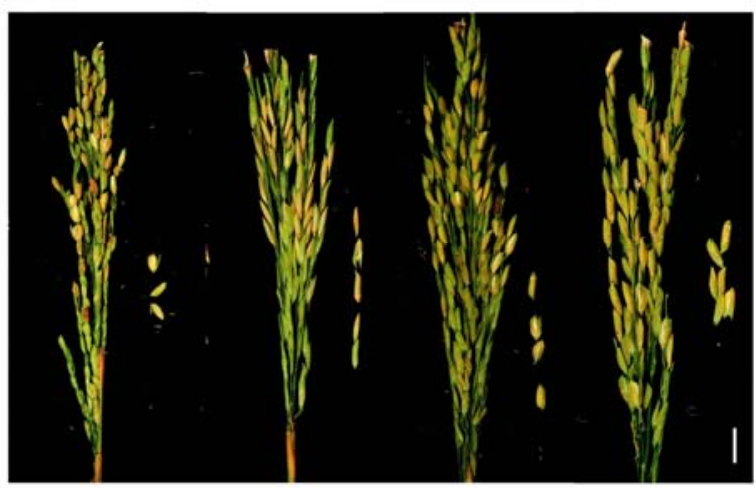

b.

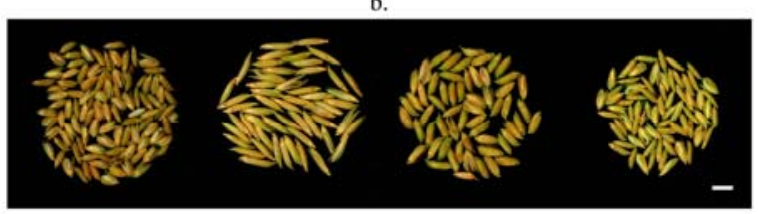

c.

Note: (a) The modified flowering stimulant (solution II) induced flowering in different male-sterile lines. From left to right in all panels: Jin-4155S, Qingxiang-1A, Yixiang-1A, and Chuannong-1A. (b) Application of the modified flowering stimulant resulted in high seed set and minimal grain shattering in the male-sterile lines. $\quad$ (c) Hybrid seed yield per panicle of the male-sterile lines. Scale bars: $20 \mathrm{~mm}$ in (a); 15 mm in (b); $10 \mathrm{~mm}$ in (c).

Figure 2 Application of the modified flowering stimulant in different male-sterile lines 


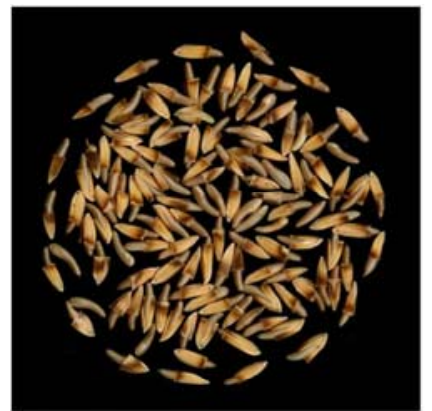

a.

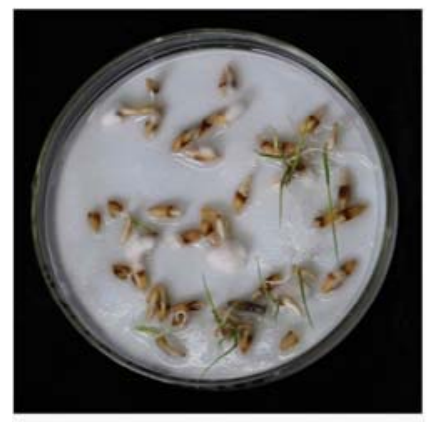

c.

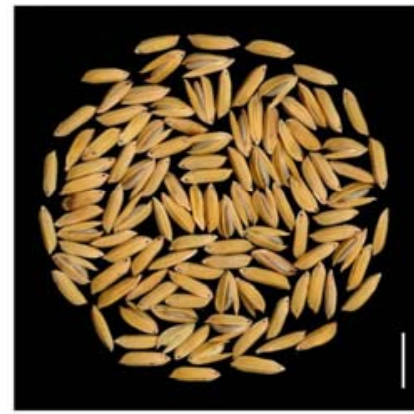

b.

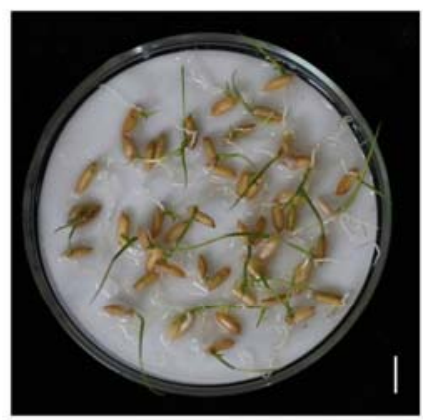

d.

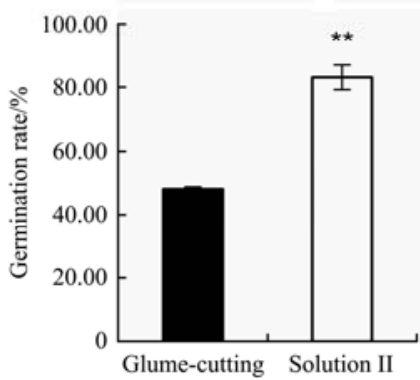

Note: (a-b) The appearance of mature hybrid seeds from glume-cut plants (a) and from plants sprayed with the flowering stimulant solution (b). (c-d) Germinated hybrid seeds from the glume-cut plants (c) and the sprayed plants (d); note that the former were more susceptible to infection. (e) Hybrid seeds from plants sprayed with the flowering stimulant solution had a higher germination rate. Scale bars: $15 \mathrm{~mm}$ in (a-b); $10 \mathrm{~mm}$ in (c-d). Values in (e) are means \pm $\mathrm{SD}(n=3)$.

Figure 3 Hybrid seeds from plants sprayed with the modified

flowering stimulant had a better appearance and a higher germination rate than hybrid seeds from glume-cut plants

\section{Discussion}

Hybrid rice technology has been a key approach for increasing rice production to meet the food demand of growing populations in China and other countries. China has the world's largest contingent of hybrid rice breeders, and there has been immense progress in rice breeding over the last 30 years. To cultivate an elite hybrid rice variety, breeders usually need to evaluate thousands of heterozygous F1 hybrids from crosses between restorer and sterile lines. The conventional preparation for these crosses involves manually cutting small spikelet glume with scissors, which requires skilled workers and is technically challenging, laborious and time consuming. At the same time, industrialization and urbanization have increased the risk of labor shortages that would negatively impact hybrid rice cultivation. Here, we developed a modified flowering stimulant that effectively induces flowering in male-sterile lines. The application of this flowering stimulant may simplify the preparation process required for rice crosses and significantly improve work efficiency. In this study, four flowering stimulant solutions were compared to evaluate their potential for use in hybrid rice cultivation (Table 1). Solution II, containing MeJA, artificial cytokinins (6-BA and KT) and Tween-20, had optimal effects on hybrid seed production and was identified as the most economical and efficient stimulant formulation (Figure 1 and Figure S1). MeJA is the active ingredient that induces spikelet flowering ${ }^{[10,12]}$, but it led to grain shattering and immature seeds when applied alone or at a very high concentration (Figure 1b and Figure S1B). These observations are consistent with the characterization of MeJA as a stress response-related phytohormone ${ }^{[8,13]}$. Cytokinins are key hormones that regulate many developmental and physiological processes in plants ${ }^{[19,20]}$, including shoot growth, fruit and seed development and delay of senescence, in addition to their roles in both abiotic and biotic stress responses. In wheat, the endogenous cytokinin level sharply increases during the phase of rapid endosperm nuclear and cell division in the developing grain, a phase critical in establishing final sink size $\mathrm{e}^{[21]}$. Similar findings were reported in rice ${ }^{[22,23]}$, indicating that cytokinins are critical for seed development and maturation. Meanwhile, the treatment of young wheat seedlings with MeJA results in a rapid and transient increase in the level of cytokinins, indicating that endogenous cytokinins promote growth and have protective effects in response to exogenous MeJA application ${ }^{[24]}$. In this study, we found that exogenous artificial cytokinins (6-BA and KT) effectively mitigated the grain shattering caused by MeJA (Figure 1b, 1c and 1e). Specifically, 6-benzylamine adenine (6-BA) and kinetin (KT) with optimal concentration were incorporated into the formulation of the flowering stimulant to mitigate the grain-shattering effect of MeJA and help ensure high hybrid seed quality.

\section{Conclusions}

The key innovation of this research is the use of synthetic cytokinins to antagonize or eliminate the adverse effects of MeJA in rice. This strategy enhances the applicability of MeJA-containing flowering stimulants for the preparation of rice crosses in hybrid rice breeding. The application of this flowering stimulant may simplify the preparation process required for rice crosses and significantly improve work efficiency.

\section{Acknowledgements}

This work was supported by the National Natural Science Foundation of China (Grant No. 31571634, 31701406) and the Foundation of the Sichuan Provincial Department of Education (Grant No. 17ZB0347).

\section{[References]}

[1] Ikeda M, Miura K, Aya K, Kitano H, Matsuoka M. Genes offering the potential for designing yield-related traits in rice. Current Opinion in Plant Biology, 2013; 16(2): 213-220.

[2] Wei X, Zhang Z, Shi P, Wang P, Chen Y, Song X, et al. Is yield increase sufficient to achieve food security in China? PLoS One, 2015; 10(2): $\mathrm{e} 0116430$.

[3] Scofield G N, Hirose T, Aoki N, Furbank R T. Involvement of the sucrose transporter, OsSUT1, in the long-distance pathway for assimilate transport in rice. Journal of Experimental Botany, 2007; 58(12): 3155-3169.

[4] Cheng S H, Zhuang J Y, Fan Y Y, Du J H, Cao L Y. Progress in research and development on hybrid rice: a super-domesticate in China. Annals of Botany, 2007; 100(5): 959-966.

[5] Huang X, Yang S, Gong J, Zhao Q, Feng Q, Zhan Q, et al. Genomic architecture of heterosis for yield traits in rice. Nature, 2016; 537(7622): 629-633.

[6] Jagadish S V, Bahuguna R N, Djanaguiraman M, Gamuyao R, Prasad P V, Craufurd P Q. Implications of high temperature and elevated $\mathrm{CO}_{2}$ on 
flowering time in plants. Frontiers in Plant Science, 2016; 7: 913.

[7] Zhong W, Wenjie G, Yizhu G. Studies on the mechanism of rice glume-opening: I Effect of $\mathrm{CO}_{2}$ on glume-opening. Acta Agronomy Sinica, 1989; 14(1): 59-66, 99. (in Chinese)

[8] Huang H, Liu B, Liu L, Song S. Jasmonate action in plant growth and development. Journal of Experimental Botany, 2017; 68(6): 1349-1359.

[9] Han G Z. Evolution of jasmonate biosynthesis and signaling mechanisms. Journal of Experimental Botany, 2017; 68(6): 1323-1331.

[10] Zeng X, Zhou X, Zhang W, Murofushi N, Kitahara T, Kamuro Y. Opening of rice floret in rapid response to methyl jasmonate. Journal of Plant Growth Regulation, 1999; 18(4): 153-158.

[11] Xiao Y, Chen Y, Charnikhova T, Mulder P P, Heijmans J, Hoogenboom A, et al. OsJAR1 is required for JA-regulated floret opening and anther dehiscence in rice. Plant Molecular Biology, 2014; 86(1-2): 19-33.

[12] Qiang Y, Hai X, Bin M, Dong G, Zheng X. Differential response of floret opening to exo-methyl jasmonate between subsp. Indica and Subsp. Japonica in Rice. Scientia Agricultura Sinica, 2014; 47(13): 2529-2540.

[13] Kim E H, Park S H, Kim J K. Methyl jasmonate triggers loss of grain yield under drought stress. Plant Signaling \& Behavior, 2009; 4(4): 348-349.

[14] Huang J Z, E Z G, Zhang H L, Shu Q Y. Workable male sterility systems for hybrid rice: Genetics, biochemistry, molecular biology, and utilization. Rice (N Y), 2014; 7(1): 13.

[15] Ouyang Y, Liu Y G, Zhang Q. Hybrid sterility in plant: stories from rice. Current Opinion in Plant Biology, 2010; 13(2): 186-192.

[16] Ding J, Lu Q, Ouyang Y, Mao H, Zhang P, Yao J, et al. A long noncoding RNA regulates photoperiod-sensitive male sterility, an essential component of hybrid rice. Proceedings of the National Academy of Sciences, USA, 2012; 109(7): 2654-2659.

[17] Xu J, Wang B, Wu Y, Du P, Wang J, Wang M, et al. Fine mapping and candidate gene analysis of ptgms2-1, the photoperiod-thermo-sensitive genic male sterile gene in rice (Oryza sativa L.). Theoretical and Applied Genetics, 2011; 122(2): 365-372.

[18] Wang S, Ma B, Gao Q, Jiang G, Zhou L, Tu B, et al. Dissecting the genetic basis of heavy panicle hybrid rice uncovered Gn1a and GS3 as key genes. Theoretical and Applied Genetics, 2018; 131(6): 1391-1403.

[19] Jameson P E, Song J. Cytokinin: a key driver of seed yield. Journal of Experimental Botany, 2016; 67(3): 593-606.

[20] Zwack P J, Rashotte A M. Interactions between cytokinin signalling and abiotic stress responses. Journal of Experimental Botany, 2015; 66(16): 4863-4871.

[21] Jameson P E, Mcwha J A, Wright G J. Cytokinins and Changes in Their Activity During the Development of Grains of Wheat (Triticum aestivum L.). Zeitschrift für Pflanzenphysiologie, 1982; 106(1): 27-36.

[22] Gu B, Zhou T, Luo J, Liu H, Wang Y, Shangguan Y, et al. An-2 Encodes a Cytokinin Synthesis Enzyme that Regulates Awn Length and Grain Production in Rice. Molecular plant, 2015; 8(11): 1635-1650.

[23] Panda B B, Sekhar S, Dash S K, Behera L, Shaw B P. Biochemical and molecular characterisation of exogenous cytokinin application on grain filling in rice. BMC Plant Biology, 2018; 18(1): 89.

[24] Avalbaev A, Yuldashev R, Fedorova K, Somov K, Vysotskaya L, Allagulova $\mathrm{C}$, et al. Exogenous methyl jasmonate regulates cytokinin content by modulating cytokinin oxidase activity in wheat seedlings under salinity. Journal of Plant Physiology, 2016; 191: 101-110. 